\title{
Anatomical Variation of Iliolumbar Artery and its Clinical Significance
}

\author{
Valchkevich Dzmitry* and Borel Anastasiya \\ Department of Normal Anatomy, Belarus
}

*Corresponding author: Valchkevich Dzmitry, Department of Normal Anatomy, Grodno State Medical University, Republic of Belarus, Belarus.
Received Date: January 29, 2019

Published Date: February 18, 2020

\section{Abstract}

The iliolumbar artery (ILA) is a standard branch from the posterior division of the internal iliac artery. The variations and branching patterns of the iliolumbar artery (ILA) and its branches are very crucial for surgeons. Iliolumbar artery has the risk of injury in pelvic trauma and regional operations. The purpose of this study was to evaluate the origin of the ILA. Iliolumbar artery was observed in 30 sides in 15 cadavers (R-15, L-15) died in the age of 55-70 years used for routine dissection. The variation of arising, some asymmetry and gender differences of ILA were established.

\section{Introduction}

The variability of the branches of the iliac arteries is described in many scientific publications dedicated to the morphological variation of their arising and topography [1-4]. However, the majority of authors study the visceral branches, but talking about the anatomy of the parietal branches of the internal iliac artery, they are not enough described in the literature. The variability in branching of the iliolumbar artery (ILA) has significant value in anatomy, medicine and in surgery, of course, because it is the very important for nutrition of the ilium bone as well as for collateral blood supply to the lower extremity in case of occlusion of the external iliac artery via anastomosis with a deep circumflex ilium artery. The ILA is used in various surgical manipulations that approach the sacroiliac joint or connections between lower lumbar vertebrae [3,4], in procedures of embolization [5-9]. Besides, variability of the iliolumbar artery needs to keep in mind in its use as feeding pedicle in bone flaps [6].

According to the majority investigations, the iliolumbar artery usually is a branch of the posterior trunk of internal iliac artery. After the beginning, the ILA passes deep under the external iliac artery on the upper one third of the iliac fossa crossing the obturator nerve $[10,11]$. Reaching the medial margin of the psoas major muscle the iliolumbar artery usually is divided into two branches. First one is the ramus lumbalis, which gives secondary branches supplying the psoas major and quadratus lumborum muscles. In most of cases, the lumbar branch anastomoses with the lumbar arteries beginning from abdominal aorta. Second branch is the iliac one. It passes deep to the iliac muscle along the medial edge of iliac crest and anastomoses with some branches including deep circumflex iliac artery beginning from the external iliac artery [12]. The deep knowledge of the anatomy of iliolumbar artery and its variability may help the surgeons to minimize the intra and postoperative complications. The aim of this study was to investigate the individual variability of iliolumbar artery.

\section{Materials and Methods}

The material of investigation was 15 cadavers of males and females died in the age of 55-70 years without pathology of vascular system. Two halves of each cadaver's pelvis were involved in research, so 30 specimens were used in total. The study was carried out with the help of dissection method, anthropometry, morphometry of blood vessels of pelvis and statistical processing (we used PC soft Statistica 10.0).

\section{Result}

Our research has shown that the iliolumbar artery with a diameter of $0.23 \pm 0.13 \mathrm{~cm}$ and a length of $2.33 \pm 1.43 \mathrm{~cm}$ in most of cases was a branch of the posterior division of the internal iliac artery (in $73.3 \%$ of cases). In $16.6 \%$, the ILA took origin from the dorsolateral surface of internal iliac artery proper. The iliolumbar 
artery had other variations more rarely. It arose from superior gluteal artery in $3.3 \%$; with the single trunk with lateral sacral artery started from the internal iliac artery in 3.3\%; with the one trunk with the obturator artery arising from the anterior trunk of internal iliac artery in $3.3 \%$.

Moreover, the sexual differences in the beginning of the iliolumbar artery were shown in the present study. It was noted that the ILA began from the superior gluteal artery only in females and only on the left half of the pelvis. The iliolumbar artery arose from the internal iliac artery more often in males (in 27.2\%) comparing females (in 10.5\%). On the contrary, the ILA took origin from the posterior division of internal iliac more often in females $(78.9 \%)$ than in males (in 63.6\%).

Besides, the current study had found the asymmetry of the ILA, which took origin from the internal iliac artery on the right side in $25 \%$ while on the left one in $11 \%$ only. The ILA arose from the posterior trunk on the left side in $66.6 \%$ but on the right side in $77.7 \%$. In males, the right ILA originated from the posterior trunk of internal iliac artery in $75 \%$ while the left one took origin from the same source in $57.1 \%$. In female, a similar arising of ILA occurs in $90.9 \%$ on the left and only in $62.5 \%$ on the right.

The iliolumbar artery passes in the superolateral direction between the obturator nerve from lumbar plexus from one side and the lateral margin of sacral plexus from another one. At the distance of $2.33 \pm 1.43 \mathrm{~cm}$ from beginning the ILA at the level of the medial edge of psoas major muscle gives off two terminal branches: lumbar branch and the iliac branch. In addition, the ILA gives branches to the lateral muscles of abdomen; the biggest nutrient branch to the ilium.

\section{Conclusion}

- The most common origin of the ILA is the posterior trunk of the internal iliac artery is (73.3\%).

- The variation in origination of iliolumbar artery has been shown.

- $\quad$ The asymmetry and sexual differences of ILA were shown in the article.

- $\quad$ The variable origins of the ILA from different branches of the internal iliac artery should take into account during the surgical interventions in the lower abdominal region to modify the surgical tactic and prevent undesired hemorrhagic complications.

\section{Acknowledgement}

None

\section{Conflict of Interest}

No conflict of interest.

\section{References}

1. Chen RS, Liu YX, Liu CB, Hu YS, Xu DC, et al. (1999) Anatomic basis of iliac crest flap pedicled on the iliolumbar artery. Surg Radiol Anat 21(2):103107.

2. Valchkevich D, Okolokulak E (2002) The iliolumbar arteries and features of their structure. Morphology 121(2-3): 34.

3. Harrington JF (2001) Far lateral disc excision at L5-S1 complicated by iliolumbar artery incursion: case report. Neurosurgery 48(6): 13771379.

4. Kiray A, Akçali O, Tayefi H, Koşay C, Ergür I (2010) Anatomical variations of the iliolumbar artery and its relation with surgical landmarks. Acta Orthop Traumatol Turc 44(6): 464-468.

5. Weatherley CR, Emran IM, Newell RL (2010) A modification of the standard midline posterior approach to interver transverse area of the lumbar spine. Ann R Coll Surg 92: 19-22.

6. Sankaranarayanan G, Rajasekhar S (2019) Anatomical variations of the principal nutrient pedicle for iliac crest graft: the ilio-lumbar artery. Surg Radiol Anat 41(1): 125-132.

7. Bleich AT, Rahn DD, Wieslander CK, Wai CY, Roshanravan SM, et al. (2007) Posterior division of the internal iliac artery: Anatomic variations and clinical applications. Am J Obstet Gynecol 197(6):658.

8. Turan Koç, Ismail Y, Gilan, Mustafa Aktekin, Zeliha Kurtoğlu, et al. (2016) Evaluation of the origin and branching patterns of the iliolumbar artery and its implications on pelvic and vertebral surgery. Saudi Med J 37(4): 457-460.

9. Lolis E, Panagouli E, Venieratos D (2011) Study of the ascending lumbar and iliolumbar veins: surgical anatomy, clinical implications and review of the literature. Ann Anat 193(6): 516-529.

10. Rusu MC, Cergan R, Demengiu D, Curcă GC, Folescu R, et al. (2010) The iliolumbar artery-anatomic considerations and details on the common iliac trifurcation. Clin Anat 23(1): 93-100.

11. Al Talalwah W, Al Dorazi SA, Soames R (2015) The origin variability of the iliolumbar artery and iatrogenic sciatica. Eur J Orthop Surg Traumatol 25 (Suppl 1): S199-204.

12. Feneis H, Dauber W (2008) Pocket atlas of human anatomy, $4^{\text {th }}$ editon, Wemding Stuttgart, Germany, pp. 264-272. 